\title{
Fiskalpolitik im Euroraum: Reformbedarf und Reformoptionen
}

Die Regierungen der Eurozone haben in der aktuellen Krise erstmals seit Jahrzehnten die Fiskalpolitik zum aktiven Gegensteuern benutzt. Allerdings sind die europäischen Institutionen und finanzpolitischen Strukturen weiter so ausgerichtet, dass in Zukunft wieder eine prozyklische Fiskalpolitik zu erwarten ist. Um auch künftig eine angemessene Reaktion auf Konjunkturschwankungen zu garantieren, müssen die Finanzströme in Europa reformiert werden - etwa über neue EU-Budgetregeln oder durch Einführung einer EU-Arbeitslosenversicherung.

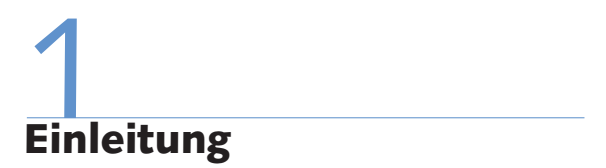

Pünktlich zum zehnten Geburtstag der Europäischen Währungsunion (EWU) erlebt die Fiskalpolitik in Europa ein überraschendes Comeback: In einer bislang unbekannten Weise versuchen alle wichtigen Euro-Länder gleichzeitig mit größeren Staatsdefiziten die schwerste Rezession der Nachkriegsgeschichte zu bekämpfen. Dabei werden nicht nur Einnahmeausfälle hingenommen, sondern auch Staatsausgaben ausgeweitet und Steuern gesenkt. Dieser Schritt wird unterstützt von Empfehlungen führender Ökonomen und Institutionen wie der Europäischen Kommission und des Internationalen Währungsfonds (IWF). Nach Prognose der Europäischen Kommission dürften die öffentlichen Defizite im Schnitt der Euro-Länder von 0,6 \% des Bruttoinlandsprodukts (BIP) im Jahr 2006 auf 5,3\% im Jahr 2009 steigen. Bis auf wenige Ausnahmen legen dabei auch die konjunkturbereinigten Defizite in fast allen Euro-Ländern deutlich zu, ein klares Zeichen fiskalpolitischer Aktivität.

Auch wenn auf aggregierter Ebene die Entwicklung der Staatshaushalte auf koordinierte Maßnahmen hindeutet, ist Europa von einer konzertierten Aktion weit entfernt: In Wirklichkeit sind die nationalen Konjunkturpakete jeweils ohne Rücksicht auf die Geschehnisse im Rest Europas geschnürt und so gestrickt, dass vorrangig die jeweilige nationale Wirtschaft profitiert. Eine inhaltliche Koordinierung oder Abstimmung der Konjunkturpakete auf europäischer Ebene gab es nicht. In Deutschland wurde die Zurückhaltung bei der konjunkturellen Stabilisierung immer wieder mit der enorm hohen Importquote insbesondere gegenüber den europäischen Partnern begründet: Die Konjunkturpakete würden „verpuffen“, weil ja ein Großteil der Kaufkraft ins Ausland abfließe. Eine auf die europäischen Bedürfnisse abgestimmte Konjunkturpolitik hätte sicher anders ausgesehen, zumal die Importe des einen EU-Landes die Exporte des anderen sind und Kaufkraft deshalb nicht durch hohe Handelsverflechtungen innerhalb Europas „verloren geht“.

Kurz: Dass die europäische Währungsunion zu einer halbwegs angemessenen fiskalpolitischen Reaktion auf die globale Wirtschaftskrise gefunden hat, hat wenig mit funktionierenden europäischen Institutionen zu tun. Unter leicht veränderten Rahmenbedingungen in einzelnen Ländern hätte die Reaktion auf die Krise deutlich schlechter ausfallen können - und wäre im Zweifel nicht im Entferntesten zeitnah und angemessen ausgefallen. Diese Vorstellung ist kein Fantasie-Gebilde: Wie weiter unten ausgeführt wird, war die Fiskalpolitik in den vergangenen zehn Jahren alles andere als optimal für die Währungsunion. Zudem haben Ökonomen in den vergangenen Jahren viel über die Bedeutung der Fiskalpolitik gelernt, wie die Beiträge in Kopcke et al. (2006) belegen.

Dieser Beitrag zeichnet die Veränderungen im Denken über die Fiskalpolitik nach (Abschnitt 2), untersucht die Entwicklungen der nationalen Fiskalpolitik seit Beginn der EWU (Abschnitt 3 und 4) und zeigt, dass die fiskalpolitischen Institutionen in Europa noch auf dem Erkenntnisstand der 1980er Jahre aufbauen. Vor dem Hintergrund der neuen Debatten werden Reformoptionen für die EWU dargestellt. Der Fokus liegt dabei auf Maßnah- men, die man ohne Komplettumbau oder eine umfangreiche Zentralisierung der Finanzbeziehungen in der Währungsunion umsetzen kann (Abschnitt 5). Ein Fazit zieht Abschnitt 6.

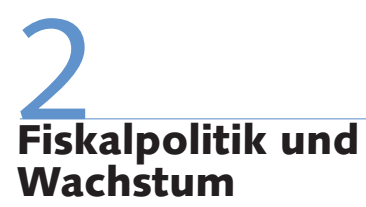

Seit dem Zweiten Weltkrieg hat sich die Bewertung der Wirkung von Fiskalpolitik durch die vorherrschende ökonomische Meinung mehrfach stark gewandelt. Während in den Jahren unter dem BrettonWoods-System fester Wechselkurse nach dem Zweiten Weltkrieg Fiskalpolitik (und vor allem das schuldenfinanzierte deficit spending) als wichtiges und wirksames Instrument nationaler Wirtschafts- und Stabilisierungspolitik angesehen wurde, fiel die Fiskalpolitik in den Jahren nach der Neuklassischen Revolution in Ungnade. Ein zentrales Element dieser von Lucas

Sebastian Dullien, Prof. Dr., lehrt Volkswirt-
schaftslehre an der Hochschule für Technik
und Wirtschaft (HTW) in Berlin. Arbeits-
schwerpunkte: Internationale Makro-
ökonomie, Europäische Wirtschafts- und
Währungsunion, Entwicklungsökonomie.
e-mail: sebastian.dullien@htw-berlin.de
Daniela Schwarzer, Dr., leitet die For-
schungsgruppen EU-Integration der Stiftung
Wissenschaft und Politik (SWP). Arbeits-
schwerpunkte: Europäische Wirtschafts- und
Währungsunion, Frankreich, Grundsatz-
fragen der Europäischen Integration.
e-mail daniela.schwarzer@swp-berlin.org

Sebastian Dullien, Prof. Dr., lehrt Volkswirtschaftslehre an der Hochschule für Technik und Wirtschaft (HTW) in Berlin. Arbeitsschwerpunkte: Internationale Makroökonomie, Europäische Wirtschafts- und Währungsunion, Entwicklungsökonomie. e-mail: sebastian.dullien@htw-berlin.de Daniela Schwarzer, Dr., leitet die Forschungsgruppen EU-Integration der Stiftung Wissenschaft und Politik (SWP). Arbeitsschwerpunkte: Europäische Wirtschafts- und Währungsunion, Frankreich, Grundsatze-mail daniela.schwarzer@swp-berlin.org 
(1973) ausgelösten Bewegung war die Idee der Ricardianischen Äquivalenz (Barro 1974). Die Neuklassiker argumentierten, dass es für Produktion und Beschäftigung keinen Unterschied mache, ob Staatsausgaben mit Schulden oder neuen Steuern finanziert würden. Rational agierende Individuen würden auf einen Anstieg der Staatsschulden mit einer Erhöhung der eigenen Ersparnis reagieren, um die künftig zur Tilgung der Staatsschulden notwendigen Steuererhöhungen bezahlen zu können. In diesem Weltbild war mithin ein Deficit Spending wirkungslos, weil der Privatsektor die zusätzliche Nachfrage des Staates durch höhere Ersparnis kompensieren würde. Zwar zweifelten eine Reihe von Studien die empirische Relevanz der Ricardianischen Äquivalenz an, die Neuklassiker erwiderten allerdings, dass der potenzielle Wohlfahrtsgewinn aus Stabilisierungspolitik zu vernachlässigen sei (Lucas 2003).

Vor diesem Erkenntnishintergrund muss auch der Maastricht-Vertrag gesehen werden, der Anfang der 1990er Jahre auf dem Höhepunkt der Erfolgswelle neuklassischen Denkens ausgehandelt wurde. Implizit enthält er (zusammen mit dem Stabilitäts- und Wachstumspakt) die Idee, dass sich die Finanzpolitik vor allem darauf konzentrieren sollte, mit der Steuerpolitik möglichst keine Anreize zu verzerren und damit langfristig ein hohes Arbeitsangebot und hohe Ersparnisse und Investitionen anzuregen. Stabilisierung durch die Finanzpolitik hat dagegen eine eher untergeordnete Rolle. So wurde auch die Begrenzung der Haushaltsdefizite auf 3,0 \% des Bruttoinlandsprodukts (BIP) mit Ausnahmen nur in Fällen dramatischer Rezessionen vom ökonomischen Mainstream als unproblematisch wahrgenommen.

In den vergangenen Jahren hat sich diese Sicht auf die Finanzpolitik wieder schleichend verändert. Die Tatsache, dass in der aktuellen Krise führende deutsche Institutionen wie der Sachverständigenrat oder die Forschungsinstitute keynesianische Konjunkturpakete ebenso befürworten (Institute 2009; Sachverständigenrat 2008) wie internationale Organisationen, ist dabei nur die Spitze einer neuen Bewegung.

Die Veränderungen sind auf drei Ebenen zu sehen: Erstens ist in der aktuellen Finanzkrise das Vertrauen in die Selbststabilisierungskräfte der Märkte geschwunden. Der globale Abschwung durch die US-Sub-
prime-Krise war derartig heftig, dass selbst konservative Institutionen wie der IWF ohne Gegensteuern ein Abrutschen in eine Abwärtsspirale wie in der großen Depression fürchteten (AFP 2008). Zweitens deutet eine Reihe von neueren Arbeiten wie etwa von Gali et al. (2005) darauf hin, dass Lucas' Schätzungen zu den Wohlfahrtsverlusten von Rezessionen deutlich zu niedrig liegen. Drittens gibt es zunehmend Anzeichen, dass man nicht - wie von der vorherrschenden Lehrmeinung lange unterstellt - den langfristigen Wachstumstrend einer Volkswirtschaft von der kurzfristigen Konjunktur trennen darf.

Dieser letzte Punkt scheint beim Design und bei der Umgestaltung der fiskalpolitischen Institutionen in der EU der wichtigste $\mathrm{zu}$ sein. Das zugrunde liegende Argument ist, dass kurzfristige Konjunkturschwankungen möglicherweise langfristige Folgen für Beschäftigung und Wirtschaftsleistung haben, dass also eine sogenannte Hysteresis auf makroökonomischer Ebene existiert (Blinder 2006). Im Kontext der neuen Wachstumstheorie argumentieren Aghion/Howitt (2006), dass übermäßige Konjunkturschwankungen Unternehmen bei der Forschungs- und Entwicklungstätigkeit einschränken: Bei imperfekten Finanzmärkten seien Unternehmen im Abschwung nicht in der Lage, ihre Forschungs- und Entwicklungsausgaben über Kredite zu finanzieren und müssen diese deshalb zurückfahren. In einem anderen Beitrag zeigen Aghion/Marinescu (2006), dass dieser Effekt statistisch signifikant ist und sich ökonometrisch nachweisen lässt. Nach den Schätzungen der Autoren könnte für Europa das Wirtschaftswachstum pro Jahr durch eine bessere Stabilisierungspolitik um bis zu einem Prozentpunkt höher ausfallen. Wenn aber starke Konjunkturschwankungen negative Folgen für das langfristige Wirtschaftswachstum haben, so müssen alle früheren Berechnungen über das Kosten-Nutzen-Verhältnis von fiskalpolitischer Stabilisierungspolitik überdacht werden. Vor allem sind dann ein neuer Ansatz der Stabilisierungspolitik und eine Reform der finanzpolitischen Institutionen in der Euro-Zone notwendige Voraussetzung, um die Lissabon-Ziele hohen Produktivitätswachstums zu erreichen.

In einer Währungsunion gewinnt die Fiskalpolitik noch einmal an Bedeutung: Nach Aufgabe der nationalen Geldpolitik bleibt den einzelnen Ländern der EWU nur die Fiskalpolitik zur Stabilisierung der nationalen Konjunkturzyklen. Zugleich spricht einiges dafür, dass die nationalen Konjunkturzyklen aus der Funktionslogik der Währungsunion verstärkt und verlängert werden (Dullien/Schwarzer 2005). Selbst wenn es der Europäischen Zentralbank auf EWU-Ebene gelingen würde, den Konjunkturzyklus perfekt zu stabilisieren, würde die Bedeutung von Stabilisierungspolitik durch Fiskalpolitik auf nationaler Ebene daher zunehmen.

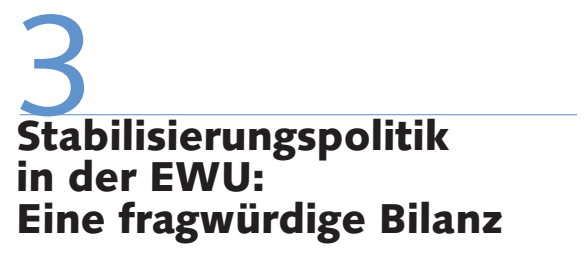

Während die Fiskalpolitik der EWU-Mitgliedstaaten in der aktuellen Krise nach Verabschiedung der enormen nationalen Konjunkturpakete für die Jahre 2009 und 2010 klar antizyklisch ausgerichtet ist, lässt sich den EWU-Ländern und der Währungsunion als Ganzes trotzdem keine effektive Stabilisierungspolitik attestieren. Die Folgen der Finanzkrise auf die Realwirtschaft sind so enorm, dass es sich um ein einmaliges „Jahrhundertereignis“ handelt. Die oben angeführten Argumente von Blinder, Aghion, Howitt und Marinescu sprechen aber für die Notwendigkeit einer Stabilisierung nicht nur in Fällen dramatischer Krisen, sondern auch in Fällen von „normalen“ Rezessionen, wie sie westliche Volkswirtschaften regelmäßig seit 1945 erlebt haben. Die folgenden Absätze analysieren deshalb die Reaktion der Finanzpolitik auf die Krise vor dem Ausbruch der Subprime-Krise 2007 und klammern den historischen Sonderfall der Jahre 2008 bis 2009 aus.

Grundsätzlich sollte man erwarten, dass die Fiskalpolitik in den Staaten Westeuropas mit ihren relativ gut ausgebauten Sozialsystemen einen wirksamen Beitrag zur Konjunkturstabilisierung leistet. Mit einer hohen Staatsquote, einem progressiven Steuersystem und relativ großzügigen Sozialleistungen sollten die automatischen Stabilisatoren stark sein. In einer detaillierten Analyse der Steuer- und Sozialsysteme errechnet van den Noord (2000), dass in den meisten EWU-Ländern eine Veränderung des BIP um $1 \%$ den Haushaltssaldo der öffentlichen Hand um etwa 0,5\% des BIP verändert, rund doppelt so stark wie in 
Tornell 1998). Für die Euro-Zone sind diese Erklärungsansätze unbefriedigend, da es nicht plausibel ist, dass der Kreditzugang schwerer sein sollte als für die USA, Großbritannien und Japan oder die politischen Institutionen schwächer und Korruption deutlich ausgeprägter sind.

\subsection{STABILISIERUNGSPOLITIK ALS ÖFFENTLICHES GUT}

Ein plausiblerer Grund ist hingegen, dass es sich bei der Zurückhaltung zu fiskalischer Stabilisierungspolitik um ein Problem der Bereitstellung eines öffentlichen Gutes handelt, als das man die Stabilisierung des Konjunkturzyklus der Euro-Zone betrachten kann: Jedes Land profitiert, die Kosten tragen aber nur jene Länder, die auch höhere Staatsschulden in Kauf nehmen. Da in der EWU die meisten Länder einen sehr hohen Offenheitsgrad haben, profitieren die Handelspartner zu einem beträchtlichen Teil von einem Konjunkturprogramm, weil dort die Exporte steigen, während die einzelne nationale Regierung die Kosten in Form von höheren Staatsschulden tragen muss. Dadurch haben besonders die Regierungen kleiner und offener Volkswirtschaften ein gedämpftes Interesse an antizyklischer Stabilisierungspolitik (Goodhart/Smith 1993, S. 423ff.). Wenn jede Regierung aufgrund der eigenen Kosten und Nutzen über fiskalpolitische Stabilisierung entscheidet, wird so in der Summe diese Stabilisierung deutlich kleiner ausfallen, als es für die Währungsunion als Ganzes optimal wäre. Dies ist auch der Grund, warum in der Literatur zum fiskalischen Föderalismus oft argumentiert wird, dass Stabilisierungspolitik auf der obersten föderalen Ebene stattfinden sollte (Collignon 2004).

\subsection{INFORMATIONSPROBLEME}

Ein weiterer Faktor könnten Informationsprobleme seitens der Regierungen sein. Eine Schwierigkeit ist, die genauen Auswirkungen der Konjunkturschwankungen auf die Einnahmen und Ausgaben des Staates abzuschätzen. In Ländern mit ausgebauten und damit komplexen Sozialsystemen, wie in Kontinentaleuropa, sind die Folgen für den Haushalt schwieriger vorherzusagen als in Ländern wie den USA, Großbritannien oder Japan mit relativ wenig ausgebauten Sozialsystemen. Die Reformen der Steuer- und Sozialsysteme in den vergan- genen Jahren haben die Schätzung von Einnahmen und Ausgaben über den Konjunkturzyklus möglicherweise noch einmal erschwert.

Überdies scheint es grundsätzlich sehr schwierig, die genaue Position im Konjunkturzyklus zu bestimmen und Wendepunkte der Konjunktur vorherzusagen. Im Frühjahr 2001, als der Abschwung in Europa schon weit fortgeschritten war, prognostizierte die Europäische Kommission für die EWU für 2001 immer noch ein Wachstum von $2,8 \%$ und für 2002 eines von $2,9 \%$. Tatsächlich wuchs die Wirtschaft nur um $1,9 \%$ und $0,9 \%$, wobei das Wachstum 2001 vor allem auf einen statistischen Überhang zurückzuführen ist. Auch die schwere Krise zur Jahreswende 2008/2009 wurde erst sehr spät wahrgenommen. Bis zum Jahresende 2008 behaupteten hochrangige Ökonomen wie DIW-Präsident Klaus Zimmermann oder der Hannoveraner Volkswirt Stefan Homburg, dass es nicht klar sei, ob die deutsche Wirtschaftsleistung 2009 tatsächlich schrumpfe. Wie wir heute wissen, befand sich die deutsche Wirtschaft bereits seit dem zweiten Quartal 2008 in der Rezession.

Ein weiteres Problem ist, dass die technischen Verfahren zur Berechnung der Produktionslücke (einem oft verwendeten Indikator zur Analyse der Position im Konjunkturzyklus) diese extrem revisionsanfällig machten: In den üblichen Verfahren hängt die aktuelle Produktionslücke stark von den Vorhersagen für die Entwicklung in den kommenden Jahren ab. Liegen die Prognosen für diese Jahre daneben, muss auch die Schätzung für die Produktionslücke revidiert werden. Diese Revisionen sind dabei alles andere als trivial: Im Herbst 2000 schätzte so die Kommission die Produktionslücke für 2000 im Euro-Raum auf minus $0,2 \%$ des BIP. Sie ging also davon aus, dass die Wirtschaft noch nicht ganz ausgelastet sei. In der Schätzung von 2005 heißt es nun, die Produktionslücke habe damals bei plus 1,7\% gelegen. Heute geht die Kommission davon aus, dass die Wirtschaft 2000 bereits fast $2 \%$ über ihrer Vollauslastung produzierte. An den tatsächlichen Wachstumszahlen für 2000 wurde indes nichts geändert. Unter solchen Vorgaben eine passende Finanzpolitik zu machen, ist praktisch unmöglich: Entweder die ursprünglichen Zahlen stimmen nicht, dann wäre die Finanzpolitik nach der neuen Schätzung unangemessen. Oder es gibt ein Problem mit der Schätzmethode (wor- auf die enorme Revisionsanfälligkeit hindeutet) und eine tatsächlich richtige Finanzpolitik wird vor dem Hintergrund dieser Daten im Nachhinein als falsch eingeschätzt. Freilich ist dies ein Problem des wissenschaftlichen Mainstreams, der bislang noch kein befriedigendes Verfahren zur Schätzung der Produktionslücke entwickelt hat. Politikern, denen der fachliche Hintergrund fehlt, um die wissenschaftliche Debatte um dieses Problem zu verfolgen, fehlt deshalb jeder verlässliche Anhaltspunkt für die Position im Konjunkturzyklus. Für die Euro-Zone stellt sich dieses Problem möglicherweise noch einmal verschärft, weil es durch die Einführung des Euro zu einer Verschiebung der Struktur der Konjunkturzyklen gekommen ist (Dullien/Schwarzer 2007).

\subsection{INSTITUTIONELLE PROBLEME DER POLITIKKOORDINIERUNG}

Man könnte natürlich argumentieren, dass die EWU über Koordinierungsmechanismen verfügt, die die Bereitstellung relevanter Informationen sichern, etwa über die wahrscheinlichen Reaktionen der makroökonomisch relevanten Akteure in der EWU (EZB, nationale und subnationale Regierungen, Sozialpartner). Darüber hinaus sollen die Regeln für die nationale Fiskalpolitik im EG-Vertrag und im Stabilitäts- und Wachstumspakt die Regierungen zu einem EWU-verträglichen Verhalten bringen. Sogenannter Peer Pressure bis hin zu Auflagen und Sanktionsdrohungen sollen die mitgliedstaatlichen Regierungen zu einem für die Währungsunion als Ganzes verträglichen Verhalten führen.

Insbesondere die auf freiwilligen Selbstverpflichtungen basierende Koordinierung, die insbesondere für Themen wie die langfristige Nachhaltigkeit und Qualität der Staatsfinanzen genutzt wird, stößt indes an ihre Grenzen: Wiederholt haben sich in der Vergangenheit die Wirtschafts- und Finanzminister in der Eurogruppe auf bestimmte Ziele geeinigt (etwa darauf, überschüssige Staatseinnahmen in die Haushaltskonsolidierung $\mathrm{zu}$ stecken), doch praktisch wurden diese Ziele nicht verwirklicht. In den meisten Fällen wurden kurzfristige politische Ziele über langfristige haushaltspolitische Erwägungen gestellt, was mit der schwachen Stellung des Finanzministers in der Regierung oder einem Regierungswechsel zusammenhängen mag (Schwarzer 2005). Es kristallisiert 
sich heraus, dass informelle Absprachen nur dann tragen, wenn die Staats- und Regierungschefs diese Ziele eindeutig mittragen.

\subsection{DIE ROLLE DES STABILITÄTS- UND WACHSTUMSPAKTS}

Überdies sind die Auswirkungen des Stabilitäts- und Wachstumspakts, der den Kern der fiskalpolitischen Regeln in der EWU darstellt, umstritten. So wird die prozyklische Wirkung der Fiskalpolitik in den Jahren 2004 und 2005 in der Mehrheit der Mitgliedstaaten von einigen Beobachtern mit dem Versuch der Regierungen begründet, die Defizitobergrenzen des Pakts in einer längeren Abschwungphase einzuhalten. Da einige Regierungen weder vor dem Abschwung 2001 bis 2003 noch vor der derzeitigen Wirtschafts- und Finanzkrise ausreichend konsolidiert hatten, war der Spielraum für das Wirken automatischer Stabilisatoren begrenzt. Die Mitgliedstaaten standen somit vor der Entscheidung, die Defizitobergrenzen zu brechen oder eine prozyklische Fiskalpolitik zu verfolgen. In Deutschland beispielsweise bezog sich die Regierung immer wieder direkt auf den Stabilitätspakt, etwa als die rot-grüne Regierung Ende 2002 beschloss, die Steuern und Sozialabgaben zum 1. Januar 2003 anzuheben. Dies geschah, obwohl die Wirtschaft immer noch am Rande einer Rezession stand und 2003 dann auch tatsächlich erneut schrumpfte. Die 3-\%-Grenze des Paktes wurde im Bundesfinanzministerium lange Zeit nicht infrage gestellt, um die Glaubwürdigkeit des Regelwerks nicht zu gefährden.

Die Kosten-Nutzen-Analyse der Bundesregierung änderte sich wohl mit dem Jahr 2004. Deutschland und Frankreich überschritten das Defizitlimit - und konnten eine Sanktionierung durch den Ecofin (Rat der Wirtschafts- und Finanzminister der EU) abwenden. Eine formelle Reform des Regelwerks zeichnete sich ab und wurde 2005 beschlossen. Sie vergrößerte den Handlungsspielraum der Regierungen, in Zeiten des Abschwungs keine prozyklische Sparpolitik verfolgen zu müssen. Dennoch zeigt die Übersicht 1 , dass gerade in diesen Jahren die Zahl der Länder mit prozyklischer Fiskalpolitik einen vorläufigen Höhenpunkt erreichte.

Dies deutet darauf hin, dass es nicht ausschließlich der Pakt war, der zuvor Anreize für eine prozyklische Sparpolitik gesetzt hatte. Neben möglichen ideologischen Vorbehalten gegen steigende Defizite könnte deshalb das oben dargestellte Argument eine Erklärung liefern, nämlich, dass einzelne Länder in einer Währungsunion mit starkem Importanteil wenig Anreize für eine antizyklische Stabilisierungspolitik im Abschwung haben. ${ }^{1}$

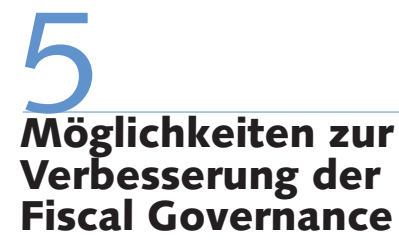

Geht man von der Prämisse aus, dass ein Totalumbau der Finanzbeziehungen innerhalb der Europäischen Währungsunion hin zu einem Föderalstaat mit sehr starker zentraler Ebene und einem entsprechenden zentralen Haushaltsvolumen wie den USA derzeit politisch unrealistisch ist, so müssen Reformoptionen gesucht werden, die die bestehenden Institutionen weiterentwickeln.

Im Folgenden werden drei institutionelle Änderungen vorgeschlagen, die darauf abzielen, auf $\mathrm{E}(\mathrm{W}) \mathrm{U}$-Ebene Elemente automatischer Stabilisierung zu verankern. Damit wird anerkannt, dass die Möglichkeit zur wirksamen konjunkturellen Stabilisierung durch nationale Haushaltspolitik unter den Bedingungen der Währungsunion mit starker Handelsverflechtung aus ökonomischen Gründen begrenzt ist und überdies an politische Umsetzungsprobleme stößt.

\subsection{EIN REGELMÄßIGER EUROZONEN-GIPFEL}

Vorab sollte dennoch überlegt werden, wie mehr politische Verbindlichkeit in die Koordinierung nationaler Haushaltspolitik in der EWU gebracht werden kann. Dies kann eine Reform des Stabilitäts- und Wachstumspakts bedeuten, würde unserer Ansicht nach aber vor allem eine Stärkung der politischen Abstimmung in der EWU erfordern. Ziel ist es, langfristig die Wahrnehmung zu fördern, dass unter den Bedingungen der EWU fiskalpolitisch an einem Strang gezogen werden sollte. Wie oben bereits ausgeführt, stoßen die freiwilligen Selbstverpflichtungen der EWUFinanzminister an Grenzen, weil diese nicht in die nationalen Politikprozesse eingebunden sind. Aus diesem Grund sollte erwogen werden, die Staats- und Regierungschefs regelmäßig mit einer Diskussion über die makroökonomischen und institutionellen Entwicklungen in der Eurozone zu befassen. Der immer wieder betonte positive Effekt der Arbeit der Eurogruppe, nämlich die Schaffung von Vertrauen und die Konsensbildung über bestehende Herausforderungen, sollten auf die oberste Entscheidungsebene ausgedehnt werden.

$\mathrm{Zu}$ diesem Zweck könnte beispielsweise dem Frühjahrsrat, der sich mit der Lissabon-Agenda und der Wettbewerbsfähigkeit der EU befasst, eine Sitzung der EWU-Länder vorgeschaltet werden. Ein zweiter längerer Eurozonengipfel sollte im Herbst eines jeden Jahres stattfinden. Der Eurozonengipfel sollte sich zum einen mit der Frage beschäftigen, inwieweit die nationalen Fiskalpolitiken nach wie vor prozyklisch wirken. Angesichts der derzeitigen Trends in den öffentlichen Staatsfinanzen im Kontext der Krise sollte auch die Nachhaltigkeit der Staatsfinanzen in den Mittelpunkt der Diskussion rücken. Dies schließt die Diskussion um eine erneute Anpassung des Regelwerks ein, mit dem Ziel, seine Glaubwürdigkeit aufrecht zu erhalten, indem die Überwachung von Risiken, die zu einer Schuldenkrise führen können (Dullien/Schwarzer 2009b) ebenso gestärkt werden wie Maßnahmen zur Verbesserung der Qualität der Staatsfinanzen.

\subsection{PROZYKLISCHE WIRKUNGEN DES EU-BUDGETS VERHINDERN}

Bei der Untersuchung prozyklischer Ausgabenpolitiken sollte das EU-Budget nicht außer Acht gelassen werden. In seiner heutigen Form hat es keine Stabilisierungsfunktion. Oft wird argumentiert, mit seiner geringen Größe von rund $1 \%$ des BIP sei ein spürbarer makroökonomischer Einfluss auch gar nicht möglich. Tatsächlich hat die Ausgabenpolitik der Gemeinschaft in der Vergangenheit in manchen Fällen prozyklisch gewirkt - mit messbaren Effekten in einigen Sektoren (Dullien/Schwarzer $2005 ; 2009 a)$.

\footnotetext{
Das Argument, dass ein deutsches Konjunkturpaket ja vor allem den Nachbarn zugutekäme, wurde übrigens erneut wiederholt von Vertretern des deutschen Finanzministeriums in der Krisenzeit 2008/2009 angeführt, um Rufe nach größeren Stützungsaktionen abzuwehren.
} 
Nach derzeitig geltendem EU-Haushaltsrecht wird ein Großteil der EU-Gelder über Jahre verplant und ohne Rücksicht auf die konjunkturelle Situation im Empfängerstaat ausgegeben. So können etwa Strukturfondsmittel zunächst einen Boom anheizen, aber genau dann abbrechen, wenn die Wirtschaft in den Abschwung gerät. Für mache Länder machen die Transfers aus dem EU-Budget einen relevanten Anteil vom BIP aus und fließen in eng abgesteckte Sektoren, sodass die Effekte spürbar sind. Für Spanien beispielsweise beliefen sich die erhaltenen Strukturfondsmittel mit 10 Mrd. $€$ von 2000 bis 2006 auf jeweils $1 \%$ des BIP. Dabei ging der größte Anteil der Transfers in den ohnehin überhitzten Bausektor.

Um eine prozyklische Wirkung des EUBudgets künftig zu verhindern, müssten einige Änderungen im Budgetprozess vorgenommen werden: Auf der Ausgabenseite könnte beispielsweise der Zeitpunkt der Mittelauszahlung von der konjunkturellen Entwicklung abhängig gemacht werden. So könnte man die Arbeit an bereits beschlossenen Infrastrukturprojekten zeitlich hinauszögern, wenn die nationale Volkswirtschaft über Trend wächst. Im Umkehrschluss würde das Geld schneller ausgegeben, wenn das tatsächliche Wachstum unter dem Trendwachstum liegt. Der Vorschlag ist dabei nicht, plötzlich die Auszahlung bewilligter Gelder einzustellen, sondern den Empfängerländern zu ermöglichen, in Absprache mit den Projektnehmern vor Ort die Spanne für die Auszahlung zu verlängern. In Zeiten etwa eines überhitzten Bausektors könnten Auftragnehmer froh über die Möglichkeit sein, Projekte nach hinten hinauszuschieben, wenn die Auftragsbücher weniger voll sind und mehr Personalressourcen zur Verfügung stehen. Eine andere, jedoch politisch schwieriger umzusetzende Variante wäre, nationale Ko-Finanzierungen der EU-geförderten Projekte entsprechend der zyklischen Situation des Empfängerlandes zu variieren. Dabei würde, wie im ersten Vorschlag, nicht die Gesamtsumme variieren, die das Land empfängt, sondern nur der Auszahlungszeitraum.

\subsection{EINE EUROPÄISCHE UNTERNEHMENSSTEUER}

Ein Beitrag zur zyklischen Stabilisierung kann auch über die Einnahmeseite des EU-Budgets geleistet werden. Dies könnte über EU-Steuern geschehen, deren Volumen sensibel auf konjunkturelle Schwankungen reagiert. Das wäre bei der Einkommensteuer oder der Unternehmenssteuer der Fall, nicht aber bei der Mehrwertsteuer. Da eine einheitliche EU-Einkommensteuer derzeit politisch nicht realisierbar ist, auch weil die einzelnen Länder sehr unterschiedliche Ansichten über den Grad der Progressivität des Steuersystems haben, scheint eine europäische Unternehmenssteuer (bei allen politischen Hürden) das realistischere Modell.

So könnte die EU über die Steuerbehörden der Mitgliedstaaten einen Steuersatz von etwa $10 \%$ auf alle Gewinne der Unternehmen in der Union einziehen, um den EU-Haushalt zu finanzieren. Dafür müssten die Einzelstaaten nicht länger den Brüsseler Etat aus den nationalen Haushalten bestreiten. Der genaue Steuersatz sollte dabei so festgelegt werden, dass die Erträge aus der Steuer bei konjunktureller Normalauslastung etwa dem heutigen EUBudget entsprechen. Jedes einzelne Land hätte darüber hinaus - wie in den USA die Einzelstaaten - die Möglichkeit, zusätzliche Steuern auf die Unternehmensgewinne zu erheben. Einige Länder würden auf diese Möglichkeit ganz verzichten, andere einen Aufschlag erheben, der eine Steuerbelastung ähnlich der aktuellen gewährleistet. Weil gleichzeitig die nationalen Budgets an anderer Stelle entlastet würden, gäbe es keinen Grund, mit einer insgesamt höheren Steuerbelastung der Unternehmen zu rechnen. Als Nebeneffekt wäre zudem ein Mindestmaß an Unternehmensbesteuerung in der EU festgeschrieben, gleichzeitig der Steuerwettbewerb nicht verhindert.

Der wichtigste Effekt wäre, dass über die Einnahmeseite eine gewisse regionale Konjunkturstabilisierung gegeben wäre: Gewinne sind üblicherweise in Boomzeiten besonders hoch. In dieser Phase würde ein Land besonders viel zum EU-Haushalt beitragen. Dafür würden die Steuereinnahmen der nationalen Regierung weniger kräftig sprudeln, was die Politiker von prozyklischen Ausgabenerhöhungen oder Steuersenkungen abhalten dürfte. In Schwächephasen dagegen fallen gewöhnlich auch die Gewinne sehr schwach aus, die Überweisungen nach Brüssel würden entsprechend zurückgehen. In dieser Situation würden die nationalen Haushalte weniger als bisher belastet, prozyklische Einsparungen würden unwahrscheinlicher.

\subsection{EINE EUROPÄISCHE ARBEITSLOSENVERSICHERUNG}

Eine dritte mögliche Säule eines EU- oder EWU-weiten Stabilisierungsmechanismus wäre eine europäische Arbeitslosenversicherung (Dullien 2008). Zwar unterscheiden sich die Systeme zur Absicherung von Arbeitslosigkeit in den einzelnen Euro-Staaten in Ausgestaltung und Leistungshöhe. Die überwiegende Zahl der Staaten erhebt aber Sozialabgaben auf die Löhne, aus der bei Arbeitslosigkeit Leistungen bezahlt werden. Ein Teil dieser Versicherung könnte auf europäische Ebene gehoben werden.

Das Problem unterschiedlicher Leistungshöhen zwischen den einzelnen Ländern ist dabei deutlich geringer, als es auf den ersten Blick erscheint: Natürlich wäre es wenig sinnvoll, wenn etwa ein portugiesischer Arbeitsloser das Arbeitslosengeld eines deutschen Facharbeiters bekommen würde, das über dem gängigen Lohn in Portugal liegt. Wie in nationalen Arbeitslosenversicherungen sollte sich die Auszahlung nach dem zuvor erzielten Einkommen richten. Um zudem zu verhindern, dass einzelne Staaten unpopuläre Reformen unterlassen, die die nationale Arbeitslosigkeit senken würden, und die Kosten auf die europäische Arbeitslosenversicherung abwälzen, sollte die maximale Bezugsdauer aus dem europäischen System auf ein Jahr begrenzt werden.

Wie auch bei der Unternehmenssteuer würde die Arbeitslosenversicherung auf EU-Ebene nicht für alle Länder gleiche Standards bedeuten. Stattdessen würde lediglich ein Mindestniveau der Absicherung geschaffen. Jeder einzelne Staat könnte über ein zusätzliches nationales System eine großzügigere Absicherung garantieren.

Das System könnte auch in Form einer Rückversicherung der nationalen Arbeitslosenversicherungen funktionieren: Die nationalen Versicherungen würden dabei einen bestimmten Anteil der nationalen Lohnsumme an die europäische Institution abführen, bei Arbeitslosigkeit der Versicherten erhält die nationale Versicherung Transfers aus Brüssel. Den nationalen Regierungen bliebe erspart, im Konjunkturabschwung Beiträge zu erhöhen oder die nationale Arbeitslosenversicherung aus dem allgemeinen Staatsbudget zu alimentieren. Im Boom würde nationale Kaufkraft abgeschöpft und über die europäische Versicherung an jene Länder ausgeschüttet, die nur langsam wachsen. 


\section{6 \\ Schlussfolgerung}

Die empirische Analyse hat gezeigt: Trotz der bestehenden Regeln und Mechanismen zur Koordinierung nationaler Fiskalpolitik hat die Fiskalpolitik in der Eurozone - sowohl auf EWU-Ebene aggregiert wie auch auf nationaler Ebene in einzelnen Mitgliedsländern - nicht stabilisierend ge- wirkt. Wir haben argumentiert, dass die meisten Maßnahmen, die dieser Tendenz entgegenwirken können, auf EU/EWUEbene angesiedelt werden sollten. So würde beispielsweise die Einführung automatischer Stabilisatoren auf EU- oder EWUEbene der prozyklischen Grundtendenz der Fiskalpolitik entgegenwirken. Kandidaten dafür sind bessere Regeln für die EUAusgaben, eine EU-Unternehmensteuer und eine europäische Arbeitslosenversicherung. Zudem sollte der Dialog der Staats- und Regierungschefs über die gemeinschaftliche Verantwortung für eine stabile wirtschaftliche Entwicklung gestärkt werden. Auch wenn viele der von uns vorgestellten Reformoptionen sehr utopisch anmuten mögen: Die aktuelle Finanzkrise, in der die Interdependenzen zwischen den Volkswirtschaften noch einmal besonders deutlich geworden sind, bietet eine gute Chance, auch diese Debatte voranzutreiben.

\section{LITERATUR}

AFP (2008): US economy shrinks as IMF warns of Great Depression, Agenturmeldung vom 23.12.

Aghion, P./Howitt, P. (2006): Joseph Schumpeter Lecture Appropriate Growth Policy: A Unifying Framework, in: Journal of the European Economic Association 4, S. 269-314

Aghion, P./Marinescu, I. (2006): Cyclical Budgetary Policy and Economic Growth: What Do We Learn from OECD Panel Data?, mimeo, Harvard University, http://www.economics.harvard.edu/faculty/aghion/papers/ Cyclical_Budgetary_Policy.pdf

Alesina, A./Tabellini, G. (2005): Why is Fiscal Policy Often Procyclical?, NBER Working Papers 11600

Barro, R. J. (1974): Are Government Bonds Net Wealth?, in: Journal of Political Economy 6, S. 1095-1117

Blinder, A. (2006): The Case Against The Case Against Discretionary Fiscal Policy, in: Kopcke, R. W./Tootell, G. M. B./Triest, R. K. (Hrsg.):

The Macroeconomics of Fiscal Policy, Cambridge, MA, et al.

Collignon, S. (2004): A Memo on the European Budget, Ifri, Paris Dullien, S. (2008): Eine Arbeitslosenversicherung für die Eurozone: Ein Vorschlag zur Stabilisierung divergierender Wirtschaftsentwicklungen in der Europäischen Währungsunion, SWP-Studie 2008/ S01

Dullien, S./Schwarzer, D. (2005): Eurozone unter Hochspannung: Die regionalen Konjunkturzyklen in der Währungsunion müssen stabilisiert werden, SWP Aktuell 21

Dullien, S./Schwarzer, D. (2007): The anti-growth bias of EMU's fiscal framework: On the pro-cyclicality of fiscal policy, paper presented at Workshop at Chatham House on November 6

Dullien, S./Schwarzer, D. (2009a): Bringing Macroeconomics into the EU Budget Debate: Why and How?, in: Journal of Common Market Studies 1, S. 153-174

Dullien, S./Schwarzer, D. (2009b): Die Eurozone braucht einen außenwirtschaftlichen Stabilitätspakt, SWP-Aktuell A27

Galí, J./Gertler, M./López-Salido, J. D. (2005): Markups, Gaps, and the Welfare Costs of Business Fluctuations, Universitat Pompeu Fabra, De- partment of Economics and Business, Working Paper 836 Galí, J./Perotti, R. (2003): Fiscal policy and monetary integration in Europe, in: Economic Policy 18, S. 534-572

Gavin, M./Perotti, R (1997): Fiscal Policy in Latin America, in: NBER Macroeconomics Annual 1997, S. 11-72

Goodhart, C. A. E./Smith, S. (1993): Stabilization, European Economy, Reports and Studies 5, S. 417-455

Institute (2009): Im Sog der Weltrezession, Gemeinschaftsdiagnose Frühjahr 2009, http://www.ifw-kiel.de/wirtschaftspolitik/

konjunkturprognosen/konjunkt/2009/gd 2009-1.pdf

Kaminsky, G. L./Reinhart, C. M./Vegh, C. A. (2004): When it Rains, it Pours: Procyclical Capital Flows and Macroeconomic Policies, NBER Working Paper 10780

Kopcke, R. W./Tootell, G. M. B./Triest R. K. (Hrsg.): (2006): The Macroeconomics of Fiscal Policy, Cambridge, MA, et al.

Lane, P. R./Tornell, A. (1998): Voracity and Growth, CEPR Discussion Paper 2001

Lucas, R. (1973): Econometric Policy Evaluation: A Critique, Vortrag auf der ersten Carnegie-Rochester Konferenz, in: Carnegie-Rochester Conference Series on Public Policy 1976, S. 19-46

Lucas, R.E. (2003): Macroeconomic Priorities, in: American Economic Review, 1, S. 1-14

Sachverständigenrat zur Begutachtung der gesamtwirtschaftlichen Entwicklung (2008): Die Finanzkrise meistern - Wachstumskräfte stärken, Jahresgutachten 2008/2009, Wiesbaden

Schwarzer, D. (2005): Transnational consensus building in EMU economic governance: Elite interaction and national preference formation, in: Kaiser, W./Starie, P. (Hrsg.): Transnationalism in the European Union, Oxford S. 210-227

Van den Noord, P. (2000): The Size and Role of Automatic Fiscal Stabilizers in the 1990s and Beyond, OECD Economics Department Working Papers 230 